\title{
Aprovechamiento integral de residuos lignocelulósicos
}

\section{Integral utilization of waste lignocellulosic}

\author{
J. A. Gallo-Corredor $*$ y R. A. Sarria-Villa \\ Departamento de Química, Universidad del Cauca, Colombia
}

\begin{abstract}
Resumen. Las especies Pinus patula y Pinus oocarpa entre otros son unas de las coníferas más cultivadas en el departamento del Cauca, su follaje contiene cierta cantidad de aceites esenciales, los cuales son usados en la industria cosmética y farmacéutica principalmente. La corteza de estas especies maderables es rica en compuestos polifenólicos, empleados en la fabricación de colorantes, resinas y compuestos con actividad antioxidante. Muchas de estas especies forestales son ricas en resinas naturales a partir de las cuales se pueden obtener resinas con aplicaciones industriales y líquidos con características aromáticas. Igualmente, el aserrín que se genera en su procesamiento puede ser empleado para producir biocombustibles y material combustible.
\end{abstract}

Palabras Claves. Resinas, Taninos, aceites esenciales, polifenoles, corteza, follaje, aserrín.

\begin{abstract}
The species Pinus patula and Pinus oocarpa among others are some of the most cultivated conifers in the Cauca Department, their foliage contains a certain amount of essential oils, which are used in the cosmetic and pharmaceutical industry mainly. The bark of these timber species is rich in polyphenolic compounds, used in the manufacture of dyes, resins and compounds with antioxidant activity. Many of these forest species are rich in natural resins from which resins with industrial applications and liquids with aromatic characteristics can be obtained. Likewise, the sawdust that is generated in its processing can be used to produce biofuels and combustible material.
\end{abstract}

Keywords. Resins, Tannins, essential oils, polyphenols, bark, foliage, sawdust.

Como citar. J. A. Gallo-Corredor y R. A. Sarria-Villa, "Aprovechamiento integral de residuos lignocelulósicos Caucanos", Jou. Cie. Ing., vol. 11, no. 1, pp. 41-49, 2019. doi: 10.46571/JCI.2019.1.4

\section{Introduction}

Una de las actividades forestales para lograr el aprovechamiento integral de especies maderables es el proceso de resinación, el cual consiste en la extracción de la resina contenida en el interior de las especies de tipo Pinus. En los departamentos del Cauca, Valle, Quindío, Caldas, Risaralda, no se tiene información respecto al sistema de resinación. La actividad de resinación en Colombia prácticamente es nula y se está desaprovechando el potencial de esta biomasa proveniente de los cultivos de Pino patula, pino oocarpa, pino tecunumani, pino Kesiya, entre otros. En el caso del follaje, aserrín y corteza son desechos lignocelulósicos que tampoco se están aprovechando en estas regiones y de los cuales se pueden extraer subproductos

\footnotetext{
* jagallo@unicauca.edu.co
} 
usados en la industria farmacéutica, jabones entre otras. La existencia de abundantes materiales lignocelulósicos, justifica la dedicación de un esfuerzo importante al desarrollo y adaptación de tecnologías tendientes a la utilización integral y racional de los mismos. En la industria papelera, el tratamiento previo que se hace a la madera para convertirla en astillas, antes de someterla al proceso de cocción, genera una gran cantidad de aserrín [1](Millett et al. 1976) [2] Varma et al., 2019. Los aserraderos de madera distribuidos en gran parte del las ciudades capitales y ciudades intermedias del suroccidente colombiano también generan gran cantidad de aserrín, causando problemas de contaminación, ocupan grandes espacios, generando riesgos potenciales de incendios, entre otros inconvenientes. Si no se resuelve adecuadamente, la destinación final de estos residuos, los efectos negativos antes mencionados seguirán aumentando. En los sitios de tala donde se procede a cortar las trozas, para cargarlas y luego llevarlas a las plantas procesadoras, queda gran cantidad de follaje y corteza de Eucaliptus grandis, P. patula, P. oocarpa. La figura 1 presenta un esquema del aprovechamiento de recursos forestales y maderables.

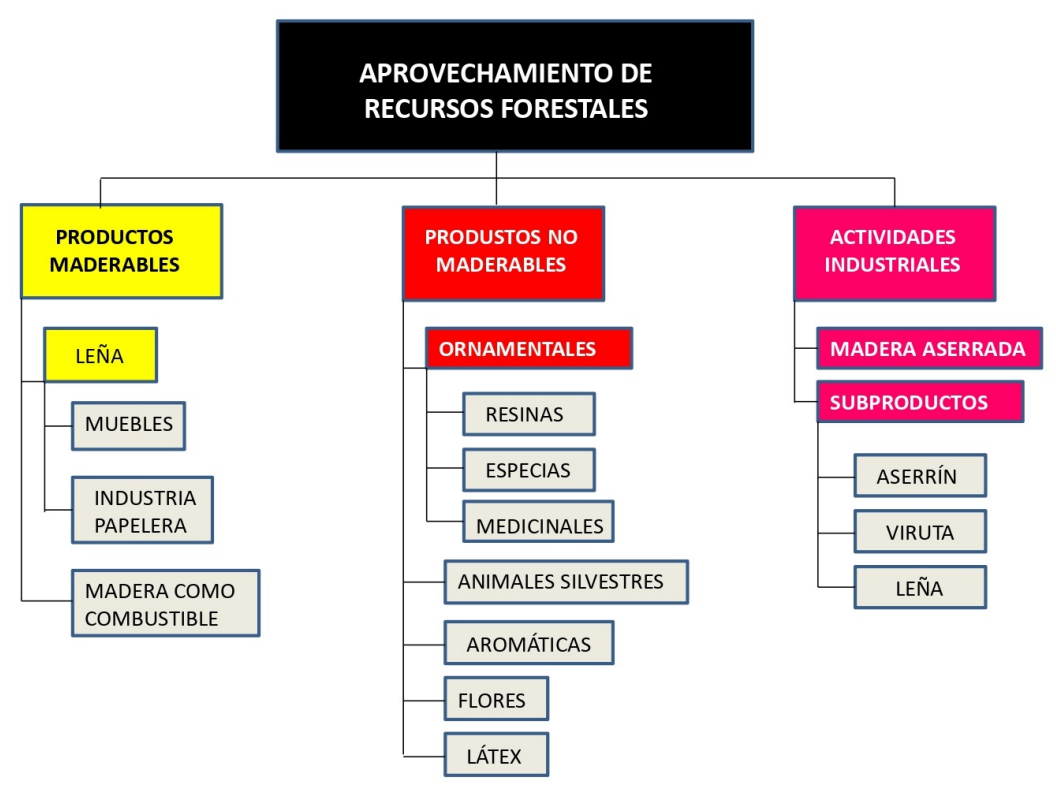

Figura 1: Aprovechamiento de recursos forestales y productos maderables 3 .

El follaje y la corteza que queda en los sitios de corte, cuando se seca puede ocasionar incendios esporádicos, la degradación natural es lenta, generando efectos negativos en su entorno y afectando finalmente las fuentes hídricas. La resina que queda adherida en las trozas de pino listas para el transporte; una vez procesadas en la planta para la obtención de la pulpa para papel, consumen gran cantidad de sustancias químicas en el proceso de deslignificación y aumenta la carga contaminante en la planta de tratamiento de agua residual. La no resinación de las variedades de Pino patula y Pino oocarpa, hacen que los subproductos obtenidos del tratamiento químico de la resina se tengan que importar [4,5]. Es importante en la región y en Colombia estudiar, organizar y orientar la fabricación de productos con valor agregado, a partir de desechos lignocelulósicos, mediante la instalación de plantas adecuadas para este fin. Actualmente se cuenta con existencias suficientes de plantaciones forestales, que garantizan la materia prima y empresas de la región decididas a obtener un valor agregado a esta actividad, disminuyendo la carga contaminante. Empresas reforestadoras de la región han prestado gran interés en este tipo de estudios en conjunto con grupos de investigación que hacen parte de instituciones de educación 
superior. El proceso de desarrollo agroindustrial de los bosques forestales continúa encadenado exclusivamente a la agroindustria del papel y del cartón, para los bosques cultivados [6], por lo cual:

- Los actores de la cadena actúan aislados.

- La producción se centra en materia prima.

- Existe baja oferta de servicios de asesoría y asistencia técnica.

- La investigación se centra en la producción de madera para pulpa.

- No existen procesos de agregación de valor.

- Se carece de un enfoque sistémico y de cadena.

\section{Aprovechamiento de los materiales lignocelulósicos y resina}

\subsection{Resina}

2.1.1. Desarrollo de la industria resinera en Colombia En el Departamento del Cauca, Valle, Quindío y Risaralda no se tiene información respecto al sistema de resinación, las variedades de pino cultivadas se utilizan principalmente en la industria del papel y para actividades de carpintería.

2.1.2. Usos de la Resina Se usa en Calefateo de los barcos, recubrimiento de piezas náuticas, azoteas de los cuartos de explosivos, elaboración de subproductos químicos, entre otros [7].

2.1.3. Composición de la resina dentro del árbol Dentro del Árbol se llama resina. Cuando es obtenida en su estado bruto se le llama miera. Una vez purificada recibe el nombre de oleoresina. De la oleoresina se le separan sus componentes sólido (Colofonia) y líquido (aguarrás). La extracción de resina se hace a través de picas o heridas al árbol vivo [8].

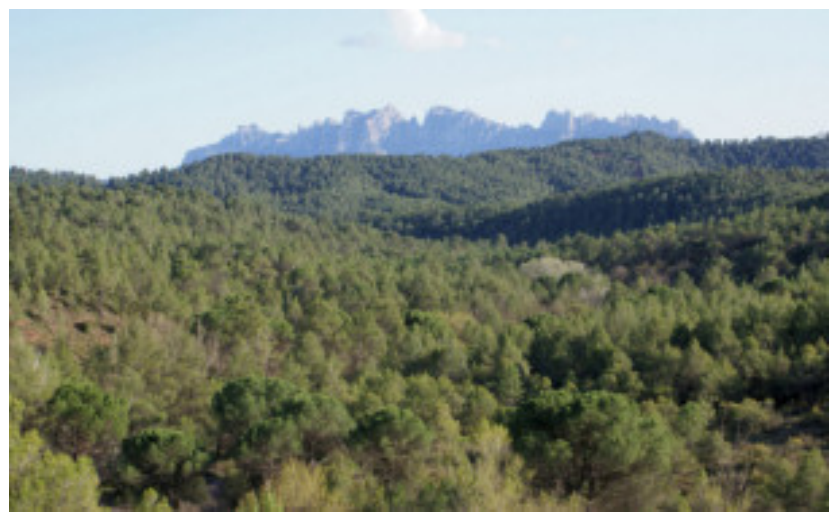

(a)

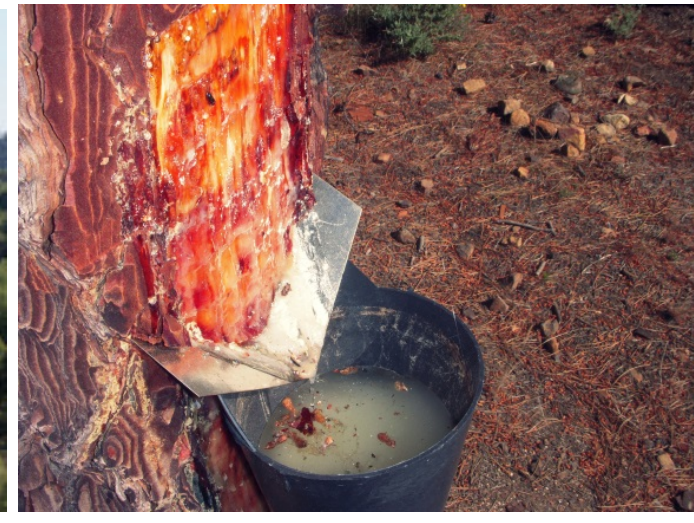

(b)

Figura 2: (a) Bosques de pino. (b) Proceso de resinación del pino.

2.1.4. Situación de la producción de la resina en Colombia Es fundamental comenzar en el sur occidente colombiano la creación de una cultura productiva relacionada con la organización, normalización, manipulación y comercialización de este producto. Iniciar la producción de un producto para la caracterización química en su primera etapa. Iniciar la producción para poder exportar segunda etapa. Y una tercera etapa para procesarla y sustituir la importación de colofonia y trementina 9 . 
2.1.5. Método de producción de resina Basándose en la información disponible hasta el momento, y contextualizándola en el departamento del Cauca, se llega a la conclusión de que el método más adecuado para las condiciones productivas de la zona, es el que se realiza practicando la extracción de la oleorresina por medio de incisiones en el árbol vivo para luego ser procesada por destilación en esencia de trementina y colofonia. El departamento del Cauca es una de las regiones con mayor explotación agroforestal en el país; cultivándose técnicamente con este propósito cerca de 9500 hectáreas de pino, principalmente de las especies P. patula y P. oоcarpa [10]. Sin desconocer las plantaciones cultivadas en Risaralda, Valle, Quindío, Caldas, Antioquia, Cundinamarca, Boyacá y Casanare. La mayor parte de estos cultivos están destinados a la producción de pulpa para papel por la empresa Smurfit Cartón de Colombia (ubicada Yumbo - Valle del Cauca), la cual cosecha los pinos después de que estos han cumplido 15 a 16 años de edad. Los árboles de pino son aptos para "resinar" después de los 12 años de edad, lo cual deja un intervalo de casi 4 años, en los que el árbol puede ser resinado, antes de su tala 11. Este es un recurso del bosque con alto potencial productivo, que hasta la fecha se está desperdiciando. En el Departamento del Cauca no existen empresas que se dediquen a la extracción y manufactura de estos productos, por lo cual la demanda nacional de estos se satisface casi en su totalidad con importaciones. Los otros métodos de producción de trementina y colofonia no son muy viables en nuestra región. El método de extracción al sulfato requeriría el montaje anterior de una productora de papel, pues este método aprovecha los vapores residuales de la cocción Kraft [11]. La remoción de los tocones de pino no es posible en nuestra región dado el alto impacto ambiental que esto generaría y la fragilidad (erosionabilidad) del suelo. Para el proceso por pirólisis de la madera requeriríamos grandes volúmenes de residuos maderables, y aunque esto sea posible, dichos residuos se aprovecharían mejor en otros procesos.

2.1.6. Capacidad de producción resinera. (Criterios y limitantes) La capacidad de producción que puede tener una planta (destiladora o fraccionadora) está determinada en principio por el volumen de materia prima disponible en la región. Este volumen depende a su ves del número de hectáreas cultivadas de pino (principalmente de la especies $P$. patula y $P$. oocarpa) aptos para resinar (con edades superiores a los 12 años). En el departamento del Cauca existen aproximadamente 3258.2 hectáreas de $P$. patula y 1465.4 hectáreas de $P$. oocarpa, para un total de 4723.6 hectáreas de pino potencialmente resinables (12 a 22 años de edad). En el Valle del Cauca existen aproximadamente 1300 hectáreas de las diferentes variedades de Pino. En el eje Cafetero (Caldas, Risaralda y Quindío) existen aproximadamente 1500 hectáreas de las diferentes variedades de Pino. En una hectárea se cultivan alrededor de 1111 pinos aproximadamente. Esta densidad de siembra puede variar según las características del terreno, la especie, y la dasonomía del bosque. Cada pino está en capacidad de producir alrededor de 1.5 litros de oleorresina en un mes. Por lo tanto una hectárea podría producir 1650 litros al mes [12].

2.1.7. Ingeniería de proceso de la resina (operación crítica) Como ya se dijo anteriormente, la materia prima a utilizar en el proceso será la oleorresina extraída por escarificación de pinos vivos. Según esto, la operación critica de nuestro proceso es básicamente una separación física de dos componentes (esencia de trementina y colofonia) presentes en una mezcla binaria (oleorresina). Esta separación se fundamenta en los diferentes grados de volatilidad que presentan los componentes de la mezcla.

\subsection{Corteza y follaje}

2.2.1. Caracterización física del follaje y corteza en coníferas El follaje y la corteza se consideran productos forestales no madereros y todos los no madereros aportan un valor agregado adicional independiente de la madera con subproductos como: Taninos, aceites esenciales, ceras, especies, condimentos, colorantes naturales, frutas, semillas entre otros. 


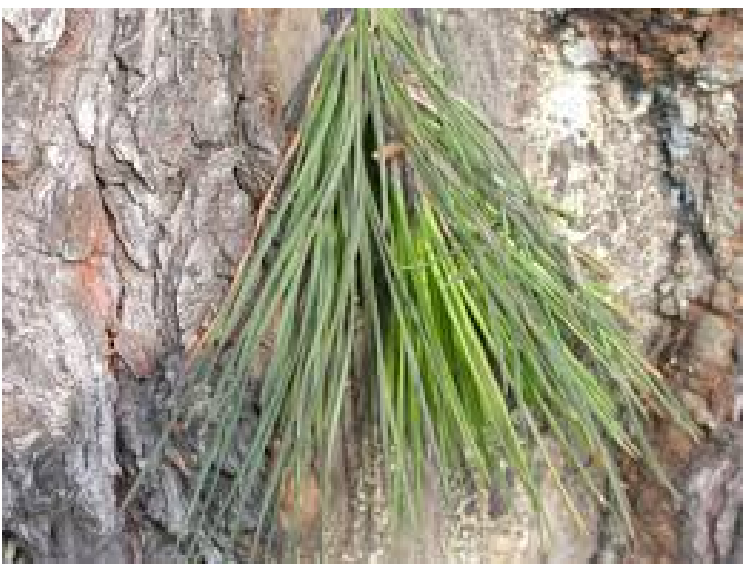

(a)

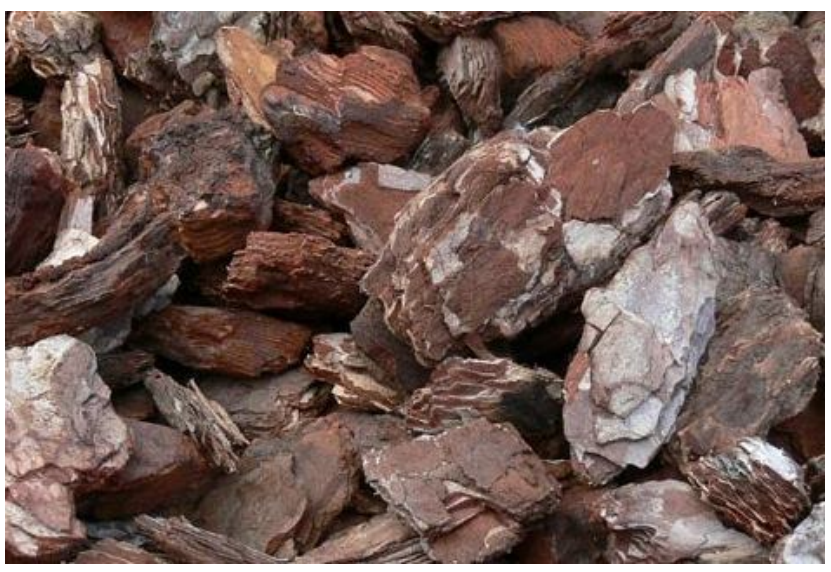

(b)

Figura 3: (a) Follaje de pino. (b) Corteza de pino. Fuente: Autores.

2.2.2. Normas para uso del follaje y corteza Partiendo primero de la caracterización física, para tal fin es fundamental la calidad del follaje y la corteza y posteriormente realizar la caracterización química de las sustancias extraíbles.

2.2.3. Obtención de productos con actividad biológica Se necesita las hojas principalmente. El contenido, de hoja buenas debe ser mayor que el $60 \%$ (calidad requerida para uso industrial. 1. categoría mayor que el $90 \%$. 2. 80 - 90\%. 3. 60 - 70\% habrá mayor \% de rendimiento si se considera la hoja). Celulosa, lignina, sustancias generales, sustancias solubles en agua, es adecuada para la obtención de determinados productos como los aceites esenciales, contenido: principalmente clorofila, carotenos 13 .

2.2.4. Composición química de la corteza Celulosa, lignina, sustancias solubles en agua, es adecuada para la obtención de determinados productos como taninos y polifenoles [14]. Minerales como oxalatos, fosfatos y silicatos han sido reportados. Calcio y potasio predominan, y trazas de boro, cobre y manganeso también se han encontrado. Entre los principales compuestos fenólicos están el ácido gálico, ácido ferúlico, ácido p-hidroxibenzoico, monómeros como la catequina, epicatequina y taxifolin y flavonoides condensados como las procianidinas 15 .

2.2.5. Aceites esenciales Los aceites esenciales son sustancias odoríferas de consistencia oleosa, generalmente líquida a temperatura y presión ordinarias, volátiles e insolubles en agua, derivados casi exclusivamente de fuentes orgánicas, en particular de los vegetales, se caracterizan por un olor pronunciado y penetrante, generalmente agradable. Las características físicas de solubilidad y volatilidad de los aceites esenciales son muy importantes, como en el caso de la preparación en perfumería donde deben utilizarse esencias que tengan características semejantes. Los aceites esenciales están formados por hidrocarburos de la serie olimentilenica del grupo de los terpenos al lado de los cuales existen otros compuestos casi siempre oxigenados, que transmiten a las esencias el aroma que las caracteriza ya que salvo algunas excepciones, los terpenos carecen de olor $[16$.

2.2.6. Estudio del transporte Cuando se acopia el follaje se debe usar lo más rápido posible debido a la degradación de pigmentos, clorofila, A. ascórbico después se da la transformación. 
Los aceites esenciales traen mayor duración. Se debe usar follaje verde o seco en estufas (pero se pueden perder sustancias). En el caso de la corteza no requiere proceso de forma inmediata [16].

\subsection{Aserrín}

La tecnología del briquetting, puede ser empleada a pequeña, mediana o gran escala. Este está clasificado como un proceso de compactación en la categoría de densificación. Tiene mucha importancia en la compactación de materiales derivados de la madera o de residuos agrícolas para la fabricación de combustibles más eficientes y de mayor poder calorífico [17]. Materiales como aserrín, cascarillas de arroz y de café, polvillo de carbón y bagazo, entre otros, han sido compactados en briquetas y utilizados como combustibles con mucho éxito. Existen dos formas básicas de briquetting: Con binder (aglomerante o "pegante") y Sin binder. Si el material no permanece unido después de prensado y se desmorona fácilmente, se hace necesaria la utilización de binder [18].

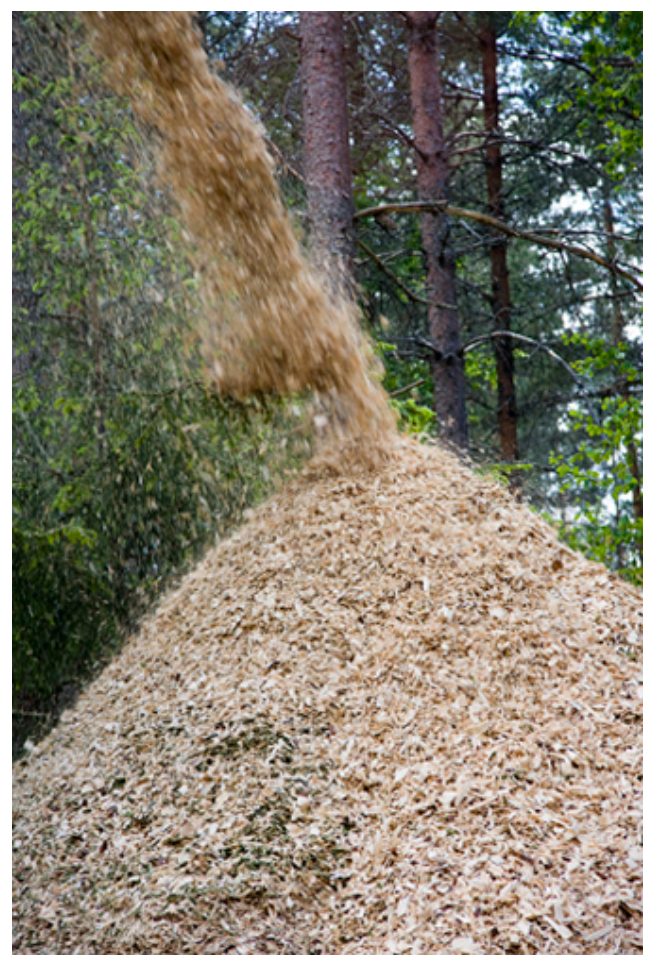

(a)

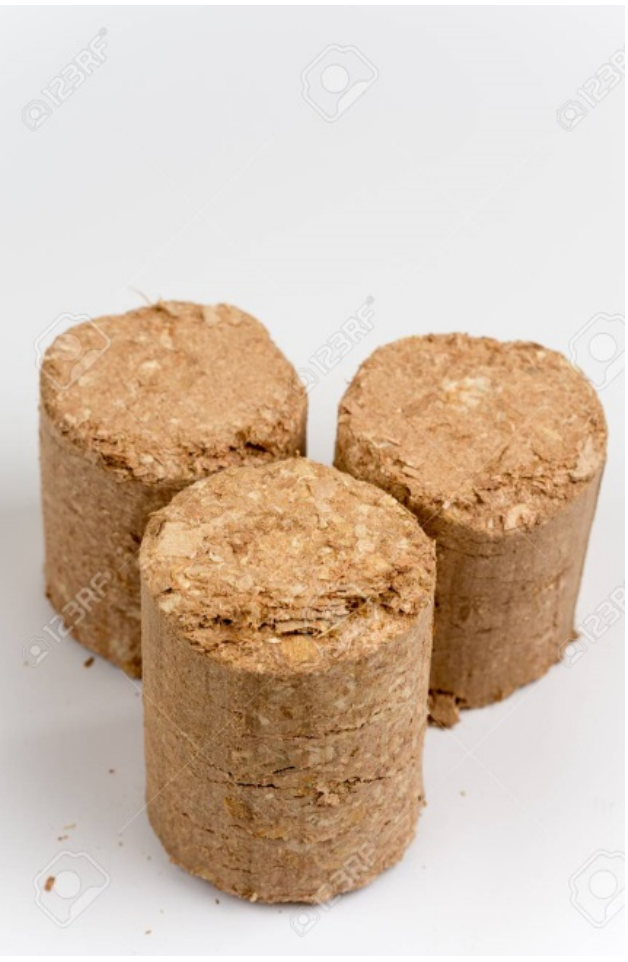

(b)

Figura 4: (a) Aserrín. (b) Briquetas de aserrín 19].

Sin embargo en algunos casos, como el aserrín, debido a las características de plasticidad que posee este material, se puede densificar sin ningún problema. En muchos lugares del mundo la fuente primaria de energía es el quemado de la madera y otros subproductos agrícolas como cascarillas de arroz y café, bagazo, cortezas y aserrín, entre otros. Estos combustibles pueden mejorar su eficiencia mediante un proceso de densificación o compresión denominado briquetting (Fabricación de briquetas) Con esta tecnología, combustibles no muy densos, como los mencionados anteriormente, se convierten en productos de mayor densidad que pueden quemarse, más fácilmente, de manera similar a la del carbón. En la industria papelera, el tratamiento previo que se hace a la madera para convertirla en astillas, antes de someterla al proceso de cocción, genera una gran cantidad de aserrín, los aserraderos de madera también generan gran cantidad de aserrín, causando problemas de contaminación, ocupan grandes 
espacios y generan un riesgo potencial de incendios, entre otros inconvenientes. Si no se resuelve, adecuadamente, la destinación final de estos residuos los efectos negativos antes mencionados seguirán aumentando. Entre las alternativas que se plantean en esta propuesta de estudio para solucionar los mencionados problemas está la utilización de aserrín como materia prima para la elaboración de briquetas que puedan utilizarse como combustible [20]. Esta tecnología ha sido ampliamente usada, desde la terminación de la segunda guerra mundial (época en la que se presentó una aguda crisis de combustibles), con mucho éxito. También es posible elaborar alcoholes y suplemento alimenticio para porcinos y equinos. Otra alternativa a explorar es la elaboración de briquetas utilizando mezclas de aserrín con lodos residuales. Este último actuaría como binder (pegante, aglomerante o aglutinante) con lo que se pretende dar uso adecuado a los tres materiales objeto de nuestra atención.

\subsection{Métodos de pretratamiento para materiales lignocelulósicos}

Existen diferentes métodos para el tratamiento de los materiales lignocelulósicos. En la tabla 1 se presentan algunos de los más importantes.

\begin{tabular}{ll}
\hline Físico & Químico \\
\hline Irradiación & Auto hidrólisis \\
Pirólisis & Explosión por Amoniaco Frío \\
Reducción de Tamaño & Oxidación Húmeda \\
Molino de Bolas & Solventes Orgánicos \\
Molino de Martillos & Oxidación $\left(\mathrm{O}_{3}, \mathrm{H}_{2} \mathrm{O}_{2}\right)$ \\
Molino de Rodillos & Gas $\left(\mathrm{SO}_{2}, \mathrm{NO}_{2}, \mathrm{ClO}_{2}\right)$ \\
& Agentes de hinchamiento $\left(\mathrm{NaOH}, \mathrm{NH}_{3}\right)$ \\
& Solventes diluyentes de la celulosa \\
& Ácido \\
& Álcali \\
\hline Físico-Químico & Biológico \\
\hline Mojado & Moho Blanco \\
Vaporizado & Bacterias \\
Explosión Por Vapor & Enzimas
\end{tabular}

Tabla 1: Métodos de pretratamiento de materiales lignocelulósicos.

Como la estructura de la celulosa es la de un polímero cristalino, asociado a una matriz con lignina y hemicelulosa y como tal es altamente resistente al ataque enzimático, un pretratamiento es por consiguiente necesario. Un pretratamiento deberá por consiguiente incrementar el número de sitios disponibles para la acción de las celulasas y promover una hidrólisis extensiva del substrato. Muchos pretratamientos resultan en un mejoramiento muy limitado en la extensión de la hidrólisis. Es importante, sin embargo, que él más efectivo pretratamiento no se juzga por la velocidad inicial de hidrólisis, sino como la celulosa hidrolizada en cualquier etapa de la reacción, puede diferir en accesibilidad y composición de la mayoría de las celulosas [21].

\section{Conclusiones}

La existencia de abundantes desechos lignocelulósicos, y el creciente problema ambiental en la región, justifica la dedicación de un esfuerzo importante al desarrollo y adaptación de tecnologías tendientes a la utilización integral y racional de los mismos. Si se obtienen estos subproductos se genera empleo directo e indirecto contribuyendo al desarrollo de la región. La disposición final de estos desechos, causan además gastos adicionales para su reubicación, 
problemas de infestación de insectos causantes de enfermedades y degradación del entorno. Con el fin de evitar problemas derivados de la poca aplicabilidad que se tiene para estos desechos, se hace necesario el desarrollo de alternativas que brinden soluciones a las empresas y comunidades, que sean amigables con el medio ambiente. La situación actual derivada de la acumulación de estos materiales residuales, requiere de una especial atención. La creciente acumulación de ellos conducirá, indefectiblemente, a causar un fuerte impacto ambiental negativo cada vez más difícil de solucionar. A esto se suma el nulo aprovechamiento que se está haciendo de un subproducto que se puede constituir en un valioso recurso energético, con muchísimas alternativas como: producción de alcohol, recuperación de sustancias volátiles, elaboración de briquetas para alimentar calderas, elaboración de suplemento alimenticio, producción de carbón vegetal, compost entre otros. Por experiencias recogidas de otros países, las alternativas han sido técnica y económicamente viables y desde el punto de vista ambiental, los resultados han sido ampliamente satisfactorios. Se estima una producción de 3 toneladas colofonia, 1 tonelada de trementina, $500 \mathrm{Kg}$ de taninos y $500 \mathrm{Kg}$ de aceites esenciales por mes tratados en una planta piloto adecuada.

\section{Agradecimientos}

Los autores agradecen a Colciencias-Colombia, al Departamento de Química de la Universidad del Cauca, a Smurfit Cartón de Colombia y a Cootraforc-Cauca-Colombia.

\section{Referencias}

[1] N. Serret-Guasch, G. Giralt-Ortega y M. Quintero-Ríos, "Caracterización de aserrín de diferentes maderas", Tecnología Química, vol. 36, no. 3, pp. 395-405, 2016.

[2] A.K. Varma, L.S. Thakur, R. Shankar, P. Mondal, "Pyrolysis of wood sawdust: Effects of process parameters on products yield and characterization of products", Waste Management, vol. 89, pp. 224-235, 2019.

[3] Aprovechamiento de recursos forestales. Disponible en: https://www youtube.com/watch?v=kZaYZ69eIBI. Consultada: 03/03/2019.

[4] J.J.W. Coppen \& G.A. Hone, "Gum naval stores: Turpentine and Rosin from pine Resin", en Food and agriculture organization of the united nations FAO, Rome, Italy, ISBN: 92-5 103584-5, 1995.

[5] C.F. de Oliveira Junkes, A.T. de Araújo Júnior, J.C. de Lima, F. de Costa, T. Füller, M. Rodrigues de Almeida, F.A. Neis, K.C. da Silva Rodrigues-Corrêa, J. Palma Fett, A.G. Fett-Neto, "Resin tapping transcriptome in adult slash pine (Pinus elliottii var. elliottii)", Industrial Crops and Products, vol. 139, no.1 pp. 111545, 2019. doi: 10.1016/j.indcrop.2019.111545.

[6] La información sobre el manejo forestal. Disponible en: http://www.fao.org/3/ad406s/AD406s05.htm. Consultada: 03/03/2019.

[7] F.A. Neis, F. de Costa, A.T. de Araújo, J. Palma Fett, A.G. Fett-Neto, "Multiple industrial uses of non-wood pine products", Industrial Crops and Products, vol. 130, pp. 248-258, 2019.

[8] J.F. Pastor-Bustamante, "Estudio de la calidad de la resina del Pinus caribaea var. caribaea y sus componentes", Revista Chapingo Serie Ciencias Forestales y del Ambiente, vol. 7, no. 2, pp. 159-162, 2001.

[9] Bosques de Pino. Disponible en: https://pinastersc.wordpress.com Consultada: 03/03/2019.

[10] Proceso de resinación. Disponible en: https://revistaecosistemasblog.net/2017/11/06/ bosques-de-pino-carrasco-que-les-depara-el-futuro/ Consultada: 04/02/2019.

[11] Z. Wang, M.M. Calderon, and M.G. Carandang, "Effects of resin tapping on optimal rotation age of pine plantation", Journal of forest economics, vol. 11, no. 4, pp. 245-260, 2006.

[12] Proexport. 2012. Sector Forestal en Colombia. Disponible en: https://www.inviertaencolombia.com.co/ Adjuntos/Perfil_Forestal_2012.pdf. Consultada: 04.02.2019.

[13] Z. Djerrad, L. Kadik, and A. Djouahri, "Chemical variability and antioxidant activities among pinushalepensiS mill. essential oils provenances, depending on geographic variation and environmental conditions", Industrial Crops and Products, vol. 74, pp. 440-449, 2015.

[14] M. Jerez, M. Pinelo, J. Sineiro, M.J. Núñez, "Influence of extraction conditions on phenolic yields from pine bark: assessment of procyanidins polymerization degree by thiolysis", Food Chemistry, vol. 94, pp. 406-414, 2006.

[15] K.J. Cho, C.H. Yun, L. Packer, A.S. Chung. "Inhibition mechanisms of bioflavonoids extracted from the 
bark of Pinus maritime on the expression of proinflammatory cytokines", Ann N Y Acad Sci. vol. 928, pp. 141-156, 2001.

[16] J.A Gallo-Corredor, R.A Sarria-Villa, "Caracterización del follaje de Pinus patula como materia prima para la extracción de aceites esenciales", Jou. Cie. Ing., vol. 7, no. 1, pp. 54-58, 2015. doi: 10.46571/JCI.2015.1.8.

[17] S.H. Mood, A.H. Golfeshan, M. Tabatabaei, G.S. Jouzani, G.H. Najafi, M. Gholami, M. Ardjmand, "Lignocellulosic biomass to bioethanol, a comprehensive review with a focus on pretreatment", Renewable and Sustainable Energy Reviews, vol. 27, pp. 77-93, 2013.

[18] B. Lela, M. Barišić, S. Nižetić, "Cardboard/sawdust briquettes as biomass fuel: Physical-mechanical and thermal characteristics", Waste Management, 47, Part B, pp. 236-245, 2016.

[19] Aserrín y briquetas. Disponible: http://alternativa-verde.com/https://es.123rf .com/photo_73345073_ tres-briquetas-de-madera-hechas-de-aserr\%C3\%ADn-prensado-.html). Consultada: 05/02/2019

[20] R.A. Sarria-Villa, J.A. Gallo-Corredor y R. Benítez-Benítez, "Condiciones óptimas de deslignificación del aserrín de Pinus patula como etapa crucial en la obtención de bioetanol", Informador Técnico, vol. 82, no. 2, pp. 160-171, 2018.

[21] W.J. Frederick, S.J. Lien, C.E. Courchene, N.A. DeMartini, A.J. Ragauskas y K. Iisa, "Co-production of ethanol and cellulose fiber from Southern Pine: a technical and economic assessment", Biomass and Bioenergy, 32, 12, pp. 1293-1302, 2008.
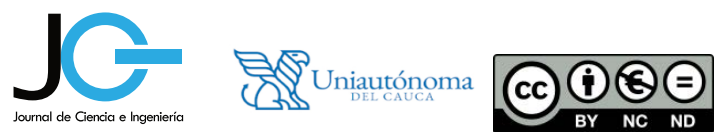